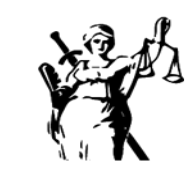

JUSTICIA

ISSN impreso 0124-7441
ISSN digital 2590-4566

\title{
Análisis conflictual de la violencia generada en las aulas de edu- cación elemental en México
}

\section{Conflict analysis of the violence generated in the classrooms of elementary education in Mexico}

\author{
Daniel Garza De La Vega \\ Universidad Autónoma de Nuevo León, México \\ mdf.dgarza@gmail.com \\ Recibido: 14 de octubre de 2019 / Aceptado: 14 de diciembre de 2019 \\ https://doi.org/10.17081/just.25.37.3457
}

\begin{abstract}
Resumen
El conflicto escolar es un problema de talla mundial y se ha buscado erradicarlo de las aulas, sin embargo pareciera que a nivel internacional esta problemática ha ido creciendo y se nota día a día en los centros escolares y en la sociedad, esta preocupación es compartida y ha llevado al estudio de programas de prevención, como lo es la mediación escolar y sus bondades para la solución de conflictos, es por ello que los agentes implicados en el conflicto deberán ser quien resuelvan sus diferencias en el mismo centro escolar. Dado esto se establece el método descriptivo y correlacional para concluir si los profesores en las aulas de la educación elemental deben estar capacitados para ser facilitadores y conciliadores en los conflictos de índole escolar.
\end{abstract}

Palabras clave: Conflicto, violencia, maestros, programas educativos, padres de familia

\begin{abstract}
The school conflict is a world-class problem and it has sought to eradicate it from the classroom, however it seems that at the international level this problem has been growing and is evident day by day in schools and in society, this concern is shared and has led to the study of prevention programs, such as school mediation and its benefits for the resolution of conflicts, that is why the agents involved in the conflict should be the one to resolve their differences in the same school. Given this, the descriptive and correlational method is established to conclude whether the teachers in elementary education classrooms should be trained to be facilitators and conciliators in school conflicts.
\end{abstract}

Keywords: Conflict, violence, teachers, educational programs, parents.

\section{Como citar:}

Garza De La Vega , D. (2020). Análisis conflictual de la violencia generada en las aulas de educación elemental en México. Justicia, 25(37), 35-48.. https://doi.org/10.17081/just.25.37.3457 


\section{Introducción}

| I conflicto escolar es una preocupación seria en la educación, se ha desbordado y transformado en varios tipos de violencia entre alumnos, maestros, padres de familia e inclusive directivos en los centros escolares, por ello que se han creado programas educativos en donde se contempla la prevención de conflictos o violencia dentro del aula.

Es preciso remarcar que los conflictos dentro de los centros escolares no son nuevos, y que cada centro cuenta con un reglamento de conducta que es regulado y modificado en base a los estatutos nacionales y estatales que los validan, sin embargo es preocupante que parecen no ser suficientes los reglamentos que rigen el comportamiento en el interior de cada centro educativo.

Hablar de conflictos en la educación es un referente diario que representa problemáticas en el interior del centro educativo, o bien cuestiones de desacuerdos o cosas que no andan bien con los alumnos, sin embargo hablar de violencia en las aulas es de preocupación de la sociedad en sí, y no solo de la misma educación ya que esto genera un ambiente de señalar culpables por la incompetencia de saber resolver problemáticas de índole agresivas.

El conflicto ha llegado a desbordar violencia en el aula por situaciones que no tienen que ver con las cuestiones escolares, la convivencia de los involucrados en los centros educativos es fundamental, si bien es cierto la escuela transmite conocimiento e intenta aplicar y formar valores, son los padres de familia los responsables que sus hijos mantengan el respeto y la cordura dentro del seno familiar y posteriormente los valores, así nos comenta " (Steele Garza 2012), "La Importancia de una de una sana convivencia en las escuelas de todos los niveles, es primordial ya que esta tiene un impacto directo con todas las personas y por ende en la sociedad. Las consecuencias de no resolver o canalizar de manera adecuada los conflictos escolares pueden ser trascendentales para la vida de los diferentes actores involucrados dentro y fuera de los recintos educativos".

Ahora bien en los últimos tiempos como ya lo hemos mencionado, se han creado programas donde se plantea la prevención de conflictos, sin embargo existe otra necesidad de resolver los conflictos entre los involucrados mediante habilidades de un facilitador que cree y adecue dentro del centro escolar un ambiente entre iguales para que los oriente hacia la solución "Si bien la problemática de la violencia escolar ha supuesto el desarrollo de programas de intervención centradas en los agresores y las victimas en las últimas décadas se ha planteado la necesidad de resolver los problemas de relaciones entre iguales" (Lopez Zafra y Berrios Martos 2005).

También unos de los factores principales que surge y se emanan de los conflictos, es la violencia, esta genera aspectos negativos dentro de los individuos hacia los demás, lastimándolos tanto física como psicológicamente.

La violencia es un mal que destruye todo a su paso, la violencia en los centros escolares es inconcebible, ya que esta no puede existir en centros de formación como son las escuelas, sin embargo la violencia en estos centros ha permeado la educación y jóvenes alumnos han sido víctimas de la misma.

Se debe comprender la violencia entre los alumnos de los centros escolares como una aberración social y todos somos responsables de erradicar esta en los centros escolares, los maestros padres de familia y directivos todos juntos serán los encargados de complementar una educación para la paz.

Según ( Prodócimo, Coelho Silva y Rodrigues Costa 2014), Existe violencia escolar cuando una persona o 
grupo de personas del centro se ve insultada, físicamente agredida, socialmente excluida o aislada, acosa$d a$, amenazada o atemorizada por otros que realizan impunemente estos comportamientos y actitudes, sintomas que se reflejan en las víctimas de violencia y que generan inseguridad y miedo en la persona afectada, así como distanciamiento notable por parte de este.

Para entender más el aspecto de violencia en las aulas las siguientes definiciones nos orientaran acerca del tema tan importante en la educación como en la sociedad:

(MÍGUEZ 2009) en cita de (D’Angelo y Fernández 2011), Nos comenta que la violencia escolar son: "situaciones extendidas de discriminación por parte del alumnado y un desinterés generalizado en las clases y en los contenidos.

(Ballester y Arnaiz Sánchez 2001), Define a la violencia como: “los problemas de la escuela desde los hechos y fenómenos que se observan desde la perspectiva de los profesores, directivos, administradores o investigadores, pero encontramos mayor dificultad en hacerlo desde las consecuencias que tiene para los alumnos la incapacidad del sistema para proporcionarles unos aprendizajes necesarios para su desarrollo personal e integración social".

(Bisquerra 2014), Define la Agresividad:- es consustancial con el ser humano y está ligada al instinto de supervivencia. La agresividad está relacionada con la defensa y en este sentido es necesaria para el ser humano.

Por su parte (Saavedra G. , Villalta P. y Muñoz 2007), Nos refiere que: “Por violencia escolar entenderemos un comportamiento coercitivo, que tiene la intención de dominar y ejercer control sobre otro sujeto (del ámbito escolar) y que se da en un contexto interpersonal, pudiendo producir daño físico, psicológico o afectar el ámbito social en cita de (Costa, M. 1998)".

Después de lo anterior surge la necesidad dentro de los centros escolares de una figura que contemple como función principal, la solución de conflictos y la generación de violencia dentro de los centros escolares.

La mediación escolar es una herramienta que se entiende como un proceso que mediante un tercero guía del mismo, va llevando para solucionar un conflicto, en los centros escolares esta herramienta sería muy útil para dirimir conflictos para no generar violencia.

Utilizar los elementos de la mediación como solución de conflictos escolares como método alterno, dirige una serie de sentimientos y comportamientos, de quienes se someten a este procedimiento para una óptima comunicación y dejar a un lado todos estos mal entendidos, que pueden generar en un tiempo considerable, daños emocionales irreparables o bien muy difíciles de cambiar en una persona.

La mediación escolar, como programa para la prevención y la resolución de conflictos dentro de la escuela, es reconocida, como un enfoque de educación para la paz que pretende una nueva imagen de los conflictos y el aprendizaje de las técnicas de análisis y regulación de conflictos de modo no violento (Fernández Herrería 1994). El aprendizaje de técnicas y el apego a los principios fundamentales de la mediación, desarrollan en el adolescente (Vazquez Gutierrez y Garcia Longoria 2013).

Existen programas preventivos que ayudan a la solución de conflictos internos, pero no han llegado a ser bastos para erradicar la problemática de violencia que se vive día a día en los centros escolares.

Dentro de los programas en México para evitar la violencia en el interior de las aulas está el que se dio a 
conocer como lo menciona (Muñoz Abundez 2008). “En febrero de 2007, se presentó el programa denominado "Escuela Segura", que pretende convertir a los centros escolares en "espacios libres de violencia, delincuencia y adicciones" (SEP, 2007). Su perspectiva es focalizarse, al menos de inicio, en los 45 municipios del país que concentran $58 \%$ de los índices delictivos, lo que implica claramente una asociación entre la conducta delictiva y las políticas de prevención de la violencia en la escuela".

\section{Qué es el conflicto escolar}

El conflicto escolar es una manifestación de la violencia en las aulas de educación, el cual involucra sentimientos negativos en quien es víctima o de quien la sufre, siempre existe una razón para la generación de este tipo de violencia que nos debe preocupar a todos ya que es un problema radicado en los centros educativos "La educación para la no violencia, que evoluciona constantemente, para esta Organización internacional no consiste sólo en negar los sentimientos de cólera o de conflicto, sino que busca canalizar la energía subyacente hacia el objetivo de elaborar estrategias eficaces y respetuosas con los otros, excluyendo las actitudes de pasividad" ( Monclús Estella 2005).

El conflicto escolar se debe a características muy específicas que no son atendidas por el profesor ya que el mismo no ha puesto su atención a sus alumnos y es muy fácil decir que el alumno se porta de manera inadecuada o que no pone la suficiente atención, el comportamiento del alumno en el aula es sumamente importante para el desarrollo de la educación "De todos es sabido que los mismos alumnos pueden comportarse de manera diferente con unos profesores o con otros. Incluso aunque la mayoría de profesores opine que determinada clase "se porta mal", la manera de comportarse mal también variará. Los alumnos tantearán y detectarán hasta donde pueden llegar: hablar en clase, interrumpir, faltar al respeto al propio profesor" (Millán Vázquez de Miguel 2001).

Podríamos clasificar los distintos conceptos de conflicto que puede haber en el ámbito educativo, sin embargo para este tema es indispensable recurrir a lo clásico, porque el conflicto en si se debe a la expresión de desigualdad entre las partes pero se da como área de oportunidad y no de confrontación así como nos dice (Vinyamata Camp 2001) "Los fundamentos de resolución de conflictos son los siguientes: el conflicto no es positivo ni negativo, el conflicto es una forma de socialización, el conflicto cumple una función social, el conflicto puede convertirse en una fuerza social positiva, ningún grupo es eternamente armonioso".

Entonces el conflicto es la falta de valores en un individuo o al parecer es lo que se percibe cuando dos o más personas ponen en riesgo la armonía que se había mantenido entre ellos, pero un conflicto cuando se lleva a su máxima expresión se origina la violencia, misma que da pie a lo inimaginable en los sentimientos personales hacia otra persona según (Rodriguez Garcia 2011) "Estaríamos ante un problema de violencia que, como define Ortega (1997), se produciría cuando: un individuo impone su fuerza, su status o su poder contra otros de forma que les ocasiona algún tipo de daño físico o psicológico, sea de forma directa o indirecta".

Dentro de la naturaleza del conflicto es indispensable llegar al fondo del mismo para determinar las causas originales, llegar al origen permitirá ver las actividades incompatibles desde el centro escolar entre los maestros, alumnos, padres de familia y comunidad que conviven a diario y es pugna expresada al menos entre las partes interdependientes que perciben objetivos incompatibles, recursos limitados y la interferencia de la otra parte en la obtención de sus objetivos. 
"En las distintas tradiciones jurídicas se ha venido distinguiendo entre dos clases de conflictos: los jurídicos y los de intereses. Para orientarse en los problemas jurídicos existe la jurisprudencia que, a partir de los casos concretos anteriores, establece una analogía entre los distintos conflictos normativos y permite ver sus similitudes y sus diferencias. No sucede lo mismo con los conflictos denominados de intereses, es decir, los no jurídicos directamente, que forman una muy variada mezcla de conflictos y frente a los cuales no existen procedimientos. $O$, en su caso, son muy insuficientes para permitir la orientación en la gestión de los mismos a quienes se enfrentan a ellos" (Redorta Lorente 2014.).

De lo anterior es necesario comentar que el conflicto atrae muchos sentimientos encontrados y emociones que se desbordan y se enfocan en la agresión para poder estallar y así manifestarse de una manera violenta como nos comenta, (Gorjón Gomez y Sáenz López 2006) "el nacimiento del conflicto es cuando surge una tensión entre las partes de una relación, esta se modifica: se pasa de armonía a la percepción del otro "legitimo otro"; se desaparece la comprensión de las emociones por necesidades del otro y la tensión se centra en que cada parte se enfoca en si misma".

El contexto y reconociendo del conflicto recae en el rol de los maestros, es preciso mostrar una situación que para ellas es motivo de preocupación e inquietud, ésta hace referencia a aquellas circunstancias, fenómenos o situaciones relacionadas con el conflicto que influyen o afectan el desarrollo del ser humano como persona individual y ciudadano perteneciente a un grupo social.

Hay ciertos tipos de conflicto como lo hemos visto, pero según (Moore 1995) "Sugiere que hay dos tipos básicos de conflictos: innecesarios y genuinos. Los conflictos innecesarios tienen como raíz problemas de comunicación y percepción, mientras que los conflictos genuinos surgen de diferencias más concretas. Según la tipología de Moore los conflictos de relaciones son innecesarios y pueden desaparecer simplemente con una comunicación apropiada y clara, mientras que los conflictos por intereses y estructurales son genuinos y su resolución requiere mayor esfuerzo".

En resumen el conflicto escolar se debe abordar y no confrontar, de la misma manera se deberá atender y estudiar desde sus perspectivas para analizar cuál es su fondo y así poder resolverlo, no haciendo caso omiso de los sentimientos de los involucrados ya que estos podrán estallar y manifestar ahora si en violencia escolar, si bien es cierto la prevención se ha incluido en los programas escolares, también es preciso la participación de simulacros de conflictos tanto como el maestro alumno y padre de familia, así nos educaremos para la solución del mismo.

\section{El conflicto escolar en México}

El conflicto escolar en México ha sufrido transformaciones muy importantes, en el Estado de Nuevo León por ejemplo la violencia que se refleja en las aulas y ha originado consecuencias irreparables, es muy grave y parece que los programas educativos donde tratan la inclusión del alumno, pareciera que no es suficiente.

Por otro lado la educación no es solo en el aula, se fomenta en casa con la familia, pero parece que la falta de convivencia familiar por la necesidad de que los padres trabajen para dar un mejor bienestar a sus familias, está originando que los niños se críen y orienten sus dudas con tecnologías mal utilizadas y televisión vaga que confunden los valores familiares dentro del hogar, "La falta de convivencia prolongada, impide que la familia pueda transmitir una serie de hábitos y valores que antes se iban adquiriendo por ese contacto prolongado de la familia y los niños" (Rodriguez Garcia 2011).

Ahora bien el conflicto escolar se ha generado en los últimos años gracias a lo dicho con anterioridad, la falta de valores en el hogar la ausencia de la educación en casa, hacen que el alumno idealice un esce- 
nario de violencia en donde el percibe que sería igual que en un programa televisivo o como en la red social donde haya tenido más interacción.

Pero, ¿es acaso la televisión o el internet el culpable del conflicto que se genera en el aula por el alumno? Como ya hemos comentado los valores son fundamentales que se enseñen en casa, el mal comportamiento de los alumnos en la escuela surge a raíz de falsos ídolos, creados principalmente por la televisión, internet y demás distractores que el alumno identifica como propios ante la falta de atención de los padres, y parece ser una costumbre con el paso del tiempo que estos niños idealicen y proyecten comportamientos agresivos no solo en las aulas sino en el hogar también "Los educadores saben que la televisión es un tema ligado fundamentalmente a la conducta, y que por tanto, los usos, los hábitos, las costumbres tienen que ver más con el ámbito familiar en el que el alumno se desenvuelve que con el ámbito escolar" (Fidalgo Yebra 2005, ).

Como ya se ha dicho, las redes sociales, la televisión y demás medios electrónicos igual que el resto del mundo, en México han sido un problema en cómo han aplicado los alumnos esta tecnología, ya que los padres han sustituido la educación de valores en el hogar por programas televisivos sin alguna supervisión de los mismos, entonces se podría decir que la televisión pasa a ser la fuente de educación de valores en el hogar ante la falta de responsabilidad de los padres, "Para Fernando Savater, la televisión es un medio de comunicación que educa demasiado y con fuerza irresistible desmitifica y disipa las nieblas de la ignorancia infantil" (Sancén Contreras 1997).

¿Hace falta mediar los conflictos? La respuesta la obtenemos cada día en las aulas, donde se genera la violencia cada día por la incapacidad de saber resolver los conflictos, parece que se dictan leyes, reformas y reglamentos para probar una disciplina en los centros escolares por parte de maestros y alumnos, misma que refleja la indisciplina por parte de las autoridades educativas para resolver esta violencia.

Habría que identificar los tipos de conflictos generados en el interior de los centros escolares para poder establecer las necesidades y esclarecer la causa principal de la violencia generada, existen tipos de reconocimiento del conflicto según (Gorjón Gomez y Sáenz López 2006), siendo estos los más importantes:

Escolar: Los niños tienen las mismas necesidades que los adultos- aprecio, amor, comprensión, alimento, sueño, aceptación- y desarrollan gran cantidad de relaciones con compañeros de escuela y maestros. Estas relaciones son susceptibles al conflicto.

De Autoestima: Es un conflicto interno que supone la autovaloración, aprobación, confianza en las capacidades personales y capacidades de sobrevivir a los fracasos. El problema surge cuando disminuye la satisfacción que el individuo siente consigo mismo. La importancia de la autoestima es tal que la Ley protege al individuo de injurias y calumnias.

De Identidad: Es el conflicto del sujeto cuando califica su conducta y no le satisface su valoración.

Así como Laborales, de Recursos escasos, de Objetivos, de Poder, de Dominación, de Competición, de Valores, de Principios, Estructural, Normativo, de Inadaptación, de Información, Atributivo, de Relaciones Personales, de Inhibición, de legitimación, de Cooperación (Gorjón Gomez y Sáenz López 2006), estos aplican para maestros, alumnos, padres de familia y ayudan a identificar los orígenes de los conflictos en su inicio.

Los conflictos son generadores de violencia, así que la generación de programas ya sean escolares, con participación de los padres de familia o de mediación dentro de los centros escolares, erradicara de golpe estos sintomas, que parecieren ser muy frecuentes en estos centros educativos y que han ocasionado prob- 
lemas de actitud y pérdida de valores éticos en la sociedad educativa.

\section{Violencia en los planteles educativos en México}

(Míguez 2007) Nos comenta los tipos de violencia que existen dentro de un campo de estudio semejante a la educación, y que estos conllevan a un enlace paralelo que unen las diferentes causas en las cuales puede originarse la violencia en las aulas, "la violencia existe también variación en lo que podríamos denominar los orígenes o causas de la violencia, podemos reconocer que paralelo a este origen pueden existir otros: la violencia delictiva, la violencia familiar, la étnica y por supuesto la escolar".

La violencia escolar contempla aspectos muy diversos para su generación como por ejemplo la falta de comunicación, la cual es un enemigo y generador de violencia por naturaleza, el erradicar de la violencia en las aulas no solo es problemática de la educación en sí, sino de la sociedad ya que esta, al generarse crea por naturaleza una conexión de inseguridades en el alumno que conlleva a una posible delincuencia prematura en el joven, exponiéndolo en un mundo hostil y de falta de oportunidades por su comportamiento antisocial.

Según (Etxeberría 2001) nos comenta acerca de la intervención europea de acuerdo a la violencia escolar "La respuesta europea ante el tema de la violencia escolar es reciente pero suficientemente rica como para poder afirmar que existe en la actualidad una base de conocimiento bastante profundo sobre determinados aspectos relacionados con el tema, tales como la dimensión del fenómeno de la violencia en la escuela, el tipo de violencia más frecuente, el distinto comportamiento de chicos y chicas, la evolución de la violencia respecto a la edad y otra serie de aspectos de importante interés".

Dentro del continente Europeo y haciendo mención que, en España al igual que muchos países sufren la violencia escolar, también han puesto en marcha desde hace ya muchos años programas de acción en contra de la misma violencia generada dentro del aula, se intenta la prevención en todo momento de conflictos que estallen en violencia generando un ambiente de paz entre los alumnos, así como nos comenta (Etxeberría 2001), "Los países europeos sufren los efectos de la violencia en distinto grado. En muchos de ellos, se ponen en marcha acciones concretas con las escuelas al objeto de reaccionar adecuadamente a situaciones de violencia y de desarrollar una prevención eficaz, capaz de promover actitudes y comportamientos positivos y pro-sociales".

De lo anterior se resume que la violencia es generada en el interior del aula por cuestiones externas, y se ve reflejado en cuestiones de alumnos padres de familia y maestros, estos últimos contribuyendo a que se genere violencia en circunstancias ya sean de índole laboral o personales, el estado es quien enfrenta este tipo de situaciones conflictivas y de violencia.

Así nos comenta (Díez-Gutiérrez 2015), "El subsidio del paro y las ayudas sociales mantienen a la gente dependiente del Estado. La gratuidad de los estudios empuja a la vagancia. Las políticas de redistribución de los beneficios desincentiva el esfuerzo", esto frente a la inconformidad de la falta de políticas educativas que generen el desabasto educativo, salarios bajos y programas educativos carentes de valores que crean vagancia en los jóvenes, dando pie a la incomprensión social como tal.

“En México, el Instituto Nacional para la Evaluación de la Educación (Aguilera, Muñoz y Orozco, 2007) ha publicado recientemente los resultados de un estudio denominado Disciplina, violencia y consumo de sustancias nocivas a la salud en escuelas primarias y secundarias de México en el que proporciona, entre otros, datos puntuales sobre la magnitud de la violencia en las escuelas primarias y secundarias del país según 
la viven y la reportan los propios alumnos y docentes. El INEE 1 incorpora a esa información lecturas sobre la manifestación de la violencia en combinación con datos de la propia escuela, de la familia y del contexto de los planteles, con base en información que proporcionaron los mismos actores" (Muñoz Abundez 2008).

\section{Mediación Escolar en México}

La mediación hace falta en las escuelas tanto como los mismos programas de prevención, la mediación es una herramienta para resolver conflictos entre los involucrados en un conflicto es por ello que es necesario un mediador que guie a través del dialogo los conflictos generados en el aula y en la escuela (nota periodística de necesidad de la mediación)².

La Mediación Escolar definida según, (García-Longoria Serrano y Vazquez Gutierrez 2013), “La mediación escolar, es el proceso de diálogo para la resolución pacífica de conflictos por el cual, las partes enfrentadas encuentran soluciones consensuadas con la intervención de una tercera persona neutral el mediador, que ayuda, facilitando el entendimiento entre las partes, a que estas soluciones sean satisfactorias y restablezcan la convivencia en las aulas" en cita de (García-Longoria y Ortuño Muñoz 2010).

Por su parte dentro de la mediación se utilizan estrategias básicas para el dialogo, el cual transforma un puente de resolución de conflictos y ayuda a transformar la violencia en áreas de oportunidad para los mediados en reconciliar su postura "La mediación puede ser considerada una estrategia en el fortalecimiento e instauración de una cultura de paz, porque auxilia a fundar un entorno pacífico ayudando a transformar los conflictos en ideas, fomenta el diálogo, la empatía, la solidaridad, la no violencia, la comprensión, la integración, la creatividad, la participación y el consenso, trascendiendo los objetivos de las partes en conflicto" (Cabello Tijerina 2013).

Como parte de los antecedentes de la mediación escolar en España ${ }^{3}$ y como nos comenta (García-Longo-

1 El Instituto Nacional para la Evaluación de la Educación (INEE) fue creado por decreto presidencial el 8 de agosto de 2002, durante el gobierno del presidente Vicente Fox Quesada, el INEE tiene como tarea principal evaluar la calidad, el desempeño y los resultados del Sistema Educativo Nacional en la educación preescolar, primaria, secundaria y media superior.

2 La mediación es una herramienta para resolver conflictos entre dos o más personas, con la intervención de alguien más que actúa en forma imparcial, es decir, un mediador. En el caso de los centros escolares, los mediadores pueden ser alumnos, profesores incluso padres que buscan satisfacer las necesidades de las partes en disputa, regulando el proceso de comunicación y conduciéndolo, si las partes colaboran voluntariamente, para llegar a una solución en la que todos queden satisfechos. Más allá de la solución a problemas interpersonales, la mediación promueve un modelo de convivencia más pacífico. En este contexto el Instituto de Justicia Alternativa del Supremo Tribunal de Justicia, recibió recientemente la solicitud de la Maestra Mirna Edith Jáquez Barranco, directora de la Escuela Secundaria Estatal Melchor Ocampo, a través del Programa Soy Legal, para que se imparta una capacitación a los maestros y maestras que forman parte de dicha escuela. La intención es impulsar mecanismos alternativos para la solución de controversias al interior del plantel y emplear la mediación para resolver conflictos relacionados con la transgresión de las normas de convivencia. Publicado por Mediación Monterrey el martes, marzo 31, 2015

3 Existe un estudio en España coordinado por una organización denominada Defensor del Pueblo avalado por la Unicef, que no necesariamente conduce a la misma conclusión. Ese trabajo cuenta con un diseño muy sólido y tiene representatividad nacional en la educación secundaria, la muestra incluyó tres mil estudiantes de 300 escuelas secundarias obligatorias; tiene la fortaleza adicional de contar con dos mediciones en el tiempo, lo que permite apreciar la evolución de la problemática en el periodo comprendido entre 1999 y 2006. Al comparar ambos estudios, uno de los resultados alentadores para España es que al parecer la tendencia en algunas manifestaciones de violencia indica una reducción con respecto de los datos disponibles de la primera mediación. Ello permite asumir que las políticas preventivas y las líneas de intervención en la escuela pueden impactar positivamente en la magnitud con la que se manifiesta esta problemática en la escuela (Muñoz Abundez 2008). 
ria Serrano y Vazquez Gutierrez 2013) “En Europa, a lo largo de los últimos 25 años el estudio de los conflictos en la escuela ha sido una cuestión prioritaria. Este hecho ha generado diversas formas de tratamiento de estos conflictos, con resultados ampliamente efectivos (Munné y Mac-Cragh 2006:19).

Según (Schroeder Q. 2004) nos comenta: Muchos países de la comunidad europea han optado por la vía de la mal llamada "Tolerancia Cero", que no es otra cosa que medidas de sentido práctico consecuentes con la necesidad básica del bien común; pues, si por culpa de algunos pocos, una inmensa mayoría no puede estudiar, se debe responsablemente buscar una salida para que las instituciones educacionales funcionen dando respuesta al clamor de la comunidad por lograr una formación de calidad para sus hijos.

La mediación escolar en España parte de experiencias aisladas llevadas a cabo por profesores pioneros con conocimientos de otras lenguas o de experiencias educativas en otros países o por grupos ya iniciados en otro tipo de conflictos no escolares, como es el caso del Centro de Resolución de Conflictos Gernika Gogoratuz. Podemos decir que la mediación escolar comienza en torno a 1993 en el País Vasco, en 1996 en Cataluña y, un año después, en 1997 en Madrid. La primera experiencia, por tanto, se produjo en el País Vasco.

(Torrego 2007) Nos indica que en 1996 comenzaron a implementarse programas de mediación en Cataluña, pero no hemos encontrado información relativa a estas primeras experiencias que parece que fueron iniciadas en algunos institutos promovidas por profesores que habían recibido formación en mediación.

En las investigaciones de la línea europea encontramos como referentes en el tema a Johan Galtung y Francisco Cascón Soriano. Este último afirma que:- "la mediación debería estar incluida dentro de un programa de educación en el conflicto en el que el objetivo fundamental fuera educar y proporcionar las herramientas necesarias para que todo el mundo pudiera ser capaz de resolver conflictos por sí mismo. Hacer lo contrario es pasar a crear una dependencia permanente de terceras partes (Educacion 2013).

Por su parte (Aznar, Cáceres y Hinojo 2007), refiere un poco al antecedente de la mediación en los países europeos y hace referencia al comportamiento social de los alumnos en España y sus conflictos, a su vez nos comenta lo siguiente: La violencia en los centros educativos ha adquirido, desde los años setenta, una magnitud apreciable en países como Estados Unidos, Suecia, Noruega y Reino Unido. Aunque la situación no es tan alarmante como en Estados Unidos.

Los mecanismos alternativos de solución de controversias surgen de una cultura pacifica de resolución de conflictos, siendo la mediación uno de ellos, la cual surge en los años 90`s y ha sido acuñada en México por el Poder Judicial como una estrategia de desahogo de los procesos judiciales y utilizadas en 21 estados de la república (Gómez 2012).

También se encuentra que la mediación es un medio para desarticular cualquier conflicto entre más de dos personas por medio de la intervención de los alumnos implicados en violencia como nos comenta (Bisquerra 2014) "Esta intervención individual se inspira en el método desarrollado por el profesor (cita de Antol Pikas 2002) denominado Shared Concern Method, que se define como una mediación terapéutica dirigida a desorganizar la estructura de dominio-sumisión que se instala entre las víctimas y agresores y que se alimenta por la presencia del grupo".

Es así que el 31 de Octubre del año 2012, con la presentación del gobierno del presidente constitucional el Lic. Enrique Peña Nieto, y el Secretario de Educación el Mtro. Emilio Chuayffet junto a él, Presidente del Tribunal Superior; asignaron un convenio de colaboración mediante el cual, maestros de todos de todos los subsistemas de educación media superior y superior, serán capacitados para utilizar la mediación escolar 
como instrumento para dirimir conflictos en los planteles educativos.

La mediación escolar, como programa para la prevención y la resolución de conflictos dentro de la escuela, es reconocida, como "un enfoque de educación para la paz que pretende un nueva imagen de los conflictos y el aprendizaje de las técnicas de análisis y regulación de conflictos de modo no violento (Fernández Herrería 1994). El aprendizaje de técnicas y el apego a los principios fundamentales de la mediación, desarrollan en el adolescente" (Vazquez Gutierrez y Garcia Longoria 2013).

Los planes y programas que se realizan para la solución de la violencia en las escuelas, quedan obsoletos día a día, sin una supervisión constante de los mismos, por ello la mediación es un método alternativo de estos reglamentos, planes y programas y es concisa, rápida y sin violencia ni resentimientos por medio de las partes.

\section{Conclusiones}

Dentro del paradigma del conflicto y su evolución a la violencia en el interior de las aulas y centros escolares, existen soluciones para erradicarlos, como hemos investigado dentro del presente artículo, la mediación escolar es fundamental para la solución de los mismos, pero obedece a un procedimiento voluntario entre las partes que se ven involucradas en violencia en las aulas, esto beneficia y motiva la solución pasiva de las problemáticas.

Dentro del marco conceptual del reconocimiento del conflicto o violencia de acuerdo sea el caso, debe existir elementos esenciales y aspectos generales las cuales el maestro, el alumno y el padre de familia al igual que el directivo deberán considerar, para reconocer la existencia de un conflicto derivado de la convivencia dentro del centro escolar.

La colaboración es esencial dentro de los centros escolares de las secundarias técnicas, ya que estos centros se manejan en conjunto y no en lo individual, es por ello que el trabajo colaborativo para el reconocimiento y la aceptación de un conflicto en común ayuda para la solución del mismo, es ahí donde se observa los directivos líderes y los maestros comprometidos con su trabajo para llevar el conflicto a una solución inmediata y concisa.

La paz y los valores son aquellos elementos anhelados por todo individuo que día a día intentamos mantener presentes en la vida cotidiana, por ello es fundamental que desde el hogar y en el aula se mantenga este paradigma "La paz no se reduce a una ausencia de guerra, fruto del equilibrio siempre precario de las fuerzas. La paz se constituye día a día, en la instauración de un orden a través de esquemas de concertación, en definitiva la paz que no surja como fruto del desarrollo integral de todos, tampoco tendrá fruto y siempre será semilla de nuevos conflictos y de variadas formas de violencia" (Gorjón Gomez 2014).

\section{Bibliografía}

Aznar, Inmaculada; Cáceres, María Pilar; Hinojo, Francisco J.,(2007), Estudio de la Violencia y Conflictividad Escolar en las Aulas de Educación Primaria a través de un Cuestionario de Clima de Clase: El Caso de las Provincias de Córdoba y Granada (España), REICE. Revista Iberoamericana sobre Calidad, Eficacia y Cambio en Educación, vol. 5, núm. 1, 2007, pp. 164-177 Red Iberoamericana de Investigación Sobre Cambio y Eficacia Escolar Madrid, España, E-ISSN: 1696-4713 RINACE@uam.es 
Díez-Gutiérrez Enrique Javier, (2015), La educación de la nueva subjetividad neoliberal, REVISTA IBEROAMERICANA DE EDUCACIÓN / REVISTA IBERO-AMERICANA DE EDUCAÇÃO vol. 68, núm. 2 (15/07/15), pp. 157172, ISSN: 1022-6508 / ISSNe: 1681-5653 Organización de Estados Iberoamericanos.

Gorjón Gómez, Francisco Javier; Sáenz López, Karla Annett Cynthia, (2006), Métodos Alternos de Solución de Controversias, Grupo Patria Cultural, primera edición, Universidad Autónoma de Nuevo León, ISBN: ISBN 970-24-0919-5.

López Zafra, Esther; Berrios Martos, M. Pilar, (2005), Violencia en las Aulas, Madrid España, Editorial DEL LUNAR, ISBN 84-95331-25-X.

Gorjón Gómez Francisco Javier, Steele Garza José Guadalupe, Acuña Zepeda Manuel Salvador, Aguilar Garnica Juan José, Vázquez Gutiérrez Reyna Lizeth ET AL, (2014) "Los MASC como atractivo de la paz y su valor intangible, Justicia en el Marco de los Derechos Humanos", La Equidad y La Justicia Alternativa Perspectiva Panameña y Mexicana, p. 275-276, Universidad Autónoma de Nuevo León, primera edición ISBN 978-607-27-0373-5

Millán Vázquez de Miguel Luis (2001), Comunicaciones al Congreso "Conflictos Escolares y Convivencia en los Centros Educativos", Secretaría General de Educación - Dirección General de Ordenación, Renovación y Centros Mérida, I.S.B.N.: 84-95251-51-5, Depósito Legal: BA-490-2001.

Monclús Estella Antonio (2005), LA VIOLENCIA ESCOLAR: PERSPECTIVAS DESDE NACIONES UNIDAS REVISTA IBEROAMERICANA DE EDUCACIÓN. N.o 38, pp. 13-32, Facultad de Educación de la Universidad Complutense, Madrid, España.

Moore, C. W. (1995). El proceso de mediación: Métodos prácticos para la resolución de conflictos. Barcelona: Garnica.

Muñoz Abundez, Gustavo, (2008), Violencia escolar en México y en otros países. Comparaciones a partir de los resultados del Instituto Nacional para la Evaluación de la Educación Revista Mexicana de Investigación Educativa, vol. 13, núm. 39, octubre-diciembre, 2008, p. 1195- 1228 Consejo Mexicano de Investigación Educativa, A.C. Distrito Federal, México, ISSN: 1405-6666.

Redorta Lorente Josep, (2014), ANÁLISIS DE CONFLICTOS POR PATRONES: LA NUEVA HERRAMIENTA CAT৫, revista Democracia Digital e Governo Eletrônico, Florianópolis, numero 10 p. 310.

Rodríguez García Pedro Luis, (2011), Análisis de la convivencia escolar en aulas de educación primaria, Revista Iberoamericana de Educación / Revista Ibero-americana de Educação, ISSN: 1681-5653 n. o - 55/3 - 15/04/11, p 1-12, Universidad de Murcia, España.

Sancén Contreras Fernando, (1997), Reseña de "El valor de educar" de Fernando Savater,

Revista Política y Cultura, núm. 9, invierno, 1997, pp. 283-287, Universidad Autónoma Metropolitana Unidad Xochimilco México, ISSN (Versión impresa): 0188-7742. Disponible en: http://www.redalyc.org/articulo.oa?id=26700915.

Steele Garza Jose Guadalupe, "Los Derechos de los consumidores, respecto a la información y publicidad en España" Molina Carrillo Julián German, Gorjón Gómez Francisco Javier, Prado Maillard José Luis, García Barrera Myrna Elia, Sáenz López Karla Annett Cynthia ET AL, (2009), Evolución del Derecho en América Latina III, p. 371, Asociación Nacional de Facultades, Escuelas, Departamentos de Derecho e Institutos de Investigación JurídicalAFEIDAL ISBN 978-607-433-218-6.

Steele Garza José Gpe, Gorjon Gómez Fco. Javier, (2012), Métodos Alternativos de solución de conflictos, Editorial Oxford, México, ISBN 978-607-426-281-0

Cabello Tijerina, Paris Alejandro, (2013), La mediación como política social. Una vía eficaz para el logro de la cultura de paz, Mediaciones Sociales, NÀ 12, 2013, pp. 191-214. ISSN electrónico: 1989-0494. DOI: 
http://dx.doi.org/10.5209/rev_MESO.2013.n12.45268, Universidad Autónoma de Nuevo León - México paris_cabello@hotmail.com

García-Longoria Serrano, María Paz y Vázquez Gutiérrez, Reyna Lizeth, La mediación escolar y las habilidades sociales en los estudiantes de educación secundaria. Un estudio en institutos de la región de Murcia, Revista: Comunitania, International Journal of Social Work and Social Sciences, 2013, enero, n. 5, pp. 113-136. http://dx.doi.org/10.5944/comunitania.5.6

Fidalgo Yebra, Manuel, (2005), Televisión y telespectadores: un conflicto permanente Revista Científica de Comunicación y Educación, núm. 25, pp. 125-130 Grupo Comunicar Huelva, España, ISSN: 1134-3478

Disponible en: http://www.redalyc.org/articulo.oa?id=15825017

Torres Merchán, Nidia Yaneth, (2010), Alternativas de solución frente a la violencia escolar Zona Próxima Revista del Instituto de Estudios en Educación Universidad del Norte, núm. 12, enero-junio, pp. 242249 Universidad del Norte Barranquilla, Colombia. Disponible en: http://www.redalyc.org/articulo. oa?id=85316155016

Schroeder Q., José Enrique (2004), La mediación escolar, un método con futuro, Pharos, vol. 11, núm. 2, noviembre-diciembre, pp. 91-96 Universidad de Las Américas Santiago, Chile, ISSN: 0717-1307, Disponible en: http://www.redalyc.org/articulo.oa?id=20811209

Gernika Gogoratuz, (1993), Experiencias de mediación escolar, 1993, España.

Ministerio de Educación, Deportes y Prevención de las Adicciones (2013), Mediación Escolar, cuadernillo practico No. 2, Programa ENREDARSE Coordinación de Mediación y Convivencia Educativa, 3a edición, Paraná, Entre Ríos Argentina.

Muñoz Abundez Gustavo, (2008), Violencia escolar en México y en otros países: comparaciones a partir de los resultados del Instituto Nacional para la Evaluación de la Educación, RMIE vol.13 no.39 México oct. /dic. 2008, versión impresa ISSN 1405-6666, disponible en http://www.scielo.org.mx/scielo. php?pid=S1405-66662008000400008\&script=sci_arttext\&tlng=pt, Revista Mexicana de Investigación Educativa (RMIE)

Gómez Yetlaneshi, (2011), Antecedentes de la Mediación en México, Programa Estatal de Mediación Escolar, disponible en.- http://programaestataldemediacion.blogspot.mx/2011/03/antecedentes-de-la-mediacion-en-mexico.html

García-Longoria Serrano, María Paz y Vázquez Gutiérrez, Reyna Lizeth, (2013), La mediación escolar y las habilidades sociales en los estudiantes de educación secundaria. Un estudio en institutos de la región de Murcia, Comunitania: International Journal of Social Work and Social Sciences № 5 / January 2013, ISSN 2173-0512

Torrego Seijo Juan Carlos, (2007). Los Conflictos En El Ámbito Educativo: Aportaciones Para Una Cultura De Paz, Primera edición: 2007, Ministerio de Educación y Ciencia, España Depósito legal: XXXX.

Schroeder Q., José Enrique, (2004), La mediación escolar, un método con futuro, Pharos, vol. 11, núm. 2, noviembre-diciembre, 2004, pp. 91-96 Universidad de Las Américas Santiago, Chile Disponible en: http://www.redalyc.org/articulo.oa?id=20811209, ISSN 0717-1307.

Bisquerra Rafael, (2014), Prevención del acoso escolar con educación emocional, editorial desclée de brouwer S.A., Bilbao España, p 57, ISBN 978-84-330-2692-7

Míguez, Daniel, (2007), Reflexiones Sobre la Violencia en el Medio Escolar Espacios en Blanco, Revista de Educación, vol. 17, junio, 2007, pp. 9-35 Universidad Nacional del Centro de la Provincia de Buenos Aires Buenos Aires, Argentina, Disponible en: http://www.redalyc.org/articulo.oa?id=384539799001, ISSN: 1515-9485. 
Luis A. D’Angelo, Daniel R. Fernández, (2011), clima, conflictos y violencia en la escuela, Fondo de las Naciones Unidas para la Infancia (UNICEF) - Facultad Latinoamericana de Ciencias Sociales (FLACSO), abril de 2011, Argentina, ISBN: 978-92-806-4599-4

Etxeberría, Félix, (2001), Europa y violencia escolar, Revista Interuniversitaria de Formación del Profesorado, núm. 41, agosto, 2001, pp. 147-165 Universidad de Zaragoza Zaragoza, España Disponible en: http:// www.redalyc.org/articulo.oa?id=27404110, ISSN: 0213-8646.

Ballester, Francisco; Arnaiz Sánchez, Pilar, (2001), Diversidad y violencia escolar Revista Interuniversitaria de Formación del Profesorado, núm. 41, agosto, 2001, pp. 39-58 Universidad de Zaragoza Zaragoza, España, Disponible en: http://www.redalyc.org/articulo.oa?id=27404104, ISSN: 0213-8646.

Saavedra G., Eugenio; Villalta P., Marco; Muñoz Q., María Teresa, (2007), Violencia escolar: la mirada de los docentes, Límite, vol. 2, núm. 15, 2007, pp. 39-60 Universidad de Tarapacá Arica, Chile, Disponible en: http://www.redalyc.org/articulo.oa?id=83601503, ISSN: 0718-1361.

Prodócimo, Elaine; Coelho Silva, Rosiane Gonçalves; Rodrigues Costa, Raquel, Violencia escolar: reflexiones sobre los espacios de ocurrencia, Revista Electrónica de Investigación Educativa, Vol. 16, Núm. 2, ISSN 1607-4041, Disponible en http://redie.uabc.mx/redie/article/view/513/922 
